\title{
Underutilization of Minimally Invasive Surgery for Colorectal Cancer
}

\author{
Steven D. Wexner, MD, FACS, FRCS, FRCS (Ed) \\ Department of Colorectal Surgery, Cleveland Clinic, Weston, FL
}

Minimally invasive surgery is underutilized for colon cancer. Since its inception in 1991, laparoscopic colorectal surgery has matured. ${ }^{1,2}$ During the nascent stages of development and dissemination of these techniques, considerations such as port site implantation led to thoughtful, measured adoption of these techniques. ${ }^{3-5}$ Moreover, the extreme technical limitations also thwarted rapid advancement. Through the slow, measured, and gradual permeation of laparoscopic colorectal surgical techniques, first for benign and then eventually for malignant conditions, surgeons improved not only their skill but also their judgment as to which patients might potentially benefit from laparoscopic colorectal surgery. Now, 20 years later, the paradigm has completely shifted: rather than trying to identify potentially suitable candidates for laparoscopic colorectal surgery, we rarely identify "routine" indications for laparotomy. Given the multifold increase in the performance of laparoscopic colorectal procedures by both general surgical and colorectal surgical residents as well as the marketing efforts of many practices, I was surprised learn that Vince et al. reported that as recently as 2005-2007, only 5.7\% of colorectal resections were performed by laparoscopy. However, their data are similar to the data reported by Lesperance et al. ${ }^{6}$ By means of similar methodology, they noted that less than $6 \%$ of all ileocolic resections for terminal ileal Crohn's disease were laparoscopically performed. Thus, perhaps it is not surprising that surgeons have even more trepidation with malignant disease. $^{6}$

It is interesting to read this number, given the fact that the statistics reported by recent graduates show that closer to $50 \%$ of their colorectal operations were laparoscopically

(C) Society of Surgical Oncology 2011

Published Online: 17 March 2011

S. D. Wexner, MD, FACS, FRCS, FRCS (Ed)

e-mail: DEANH@ccf.org undertaken. ${ }^{7}$ The main reason for this large discrepancy in this retrospective data sample versus the prospective data collection by graduating general and colorectal surgical residents is clearly stated by the authors in both their Methods and Discussion sections. "Because there were no specific codes to identify laparoscopic colonic prior to 2008, colonic resections were defined as minimally invasive if the ICD-9-CM procedure code for laparoscopy (54.21) was identified among the primary procedures in addition to a code for colectomy. All other colectomies were considered open" I believe that this methodology is flawed in that many surgeons would have been fearful of "unbundling" and adding a laparoscopic code to a colectomy procedure.

Accordingly, the potential for significant undercoding could have easily led to what the authors quite appropriately state in their discussion-specifically that this methodology "may have resulted in an under estimation of laparoscopic cases for colon cancer resection." Therefore, not only might the adoption rate have been higher, but all of the results may have been different if more of the laparoscopically performed procedures had been coded in that manner and thus included for data analysis within the laparoscopic group. There are some other, perhaps less major issues in the study, such as, as the authors also note, failure to collect body mass index data for comparison between the two groups. Moreover, features such as tumor size and location are not included, nor are potentially relevant issues in the discussion-making process, such as prior laparotomy. Unfortunately, the definition of a teaching hospital is not provided, nor are definitions offered for the Table 2 headings of bed size "small" versus "medium" versus "large." The authors found a reference to define a "high-volume" colon surgeon as someone who performs just over one procedure per month, with a reported median actually being only one case slightly less than 6 weeks. Specifically, they defined a high-volume colon surgeon as 
someone who performs at least 15 cases annually. I would consider this number to be unacceptably low to qualify as high volume, and urge the authors to consider other data sources to set their definition of a high-volume colon and rectal surgeon. ${ }^{8-12}$ The authors should reconsider the definition, which may greatly alter the results of their univariate and multivariate analyses. It has been well demonstrated within the rectal cancer literature that outcomes are indeed related to surgical volume, but the highvolume numbers must be indeed truly high volume..$^{8-14}$ Another factor of this study to be considered is that the authors do correctly reference publications that cite the learning curve for laparoscopic colorectal resection as ranging from 30 to 70 cases. ${ }^{15-18}$ However, the authors do not account for the fact that general and colorectal surgical residents, and perhaps surgical oncology and minimally invasive surgical trainees, may easily perform this requisite number during training. The authors do not detail the potential effects of cases performed during training, nor do they mention the more recently widely available training methods of simulation. ${ }^{19,20}$

The shortcomings of this article may be easily overcome when these authors or other investigators next analyze these data because laparoscopic colectomy codes have been in existence for the last several years. I suspect that we will see adoption in at least $30 \%$ of cases, if not closer to $60 \%$, at least for colon resections. The last frontier of laparoscopic colorectal surgery is proctectomy for cancer. Although this procedure may be performed by numerous individuals in an "off-label" setting, the American College of Surgeons Oncology Group Z6051 randomized, controlled trial lead by Dr. James Fleshman will, I hope, soon lead us to a data-driven answer. ${ }^{21}$ Similarly, the COLOR2 trial has been designed to assess the safety and feasibility of laparoscopic proctectomy for cancer. ${ }^{22}$

\section{REFERENCES}

1. Cooperman AM, Katz V, Zimmon D, et al. Laparoscopic colon resection: a case report. J Laparoendosc Surg. 1991;1:221-4.

2. Jacobs M, Verdeja JC, Goldstein HS. Minimally invasive colon resection (laparoscopic colectomy). Surg Laparosc Endosc. 1991;1:144-50.

3. Jacquet P, Averbach AM, Jacquet N. Abdominal wall metastasis and peritoneal carcinomatosis after laparoscopic-assisted colectomy for colon cancer. Eur J Surg Oncol. 1995;21:568-70.

4. Fusco MA, Paluzzi MW. Abdominal wall recurrence after laparoscopic-assisted colectomy for adenocarcinoma of the colon. Report of a case. Dis Colon Rectum. 1993;36:858-61.
5. Wexner SD, Cohen SM. Port site metastases after laparoscopic colorectal surgery for cure of malignancy. Br J Surg. 1995;82: $295-8$.

6. Lesperance K, Martin MJ, Lehmann R, et al. National trends and outcomes for the surgical therapy of ileocolonic Crohn's disease: a population-based analysis of laparoscopic vs. open approaches. J Gastrointest Surg. 2009;13:1251-9.

7. Schoetz DJ Jr. Evolving practice patterns in colon and rectal surgery. J Am Coll Surg. 2006;203:322-7.

8. Porter GA, Soskolne CL, Yakimets WW, et al. Surgeon-related factors and outcome in rectal cancer. Ann Surg. 1998;227: 157-67.

9. Harmon JW, Tang DG, Gordon TA, et al. Hospital volume can serve as a surrogate for surgeon volume for achieving excellent outcomes in colorectal resection. Ann Surg. 1999;230:404-11.

10. Smith JA, King PM, Lane RH, et al. Evidence of the effect of "specialization" on the management, surgical outcome and survivalfrom colorectal cancer in Wessex. Br J Surg. 2003;90: 583-92.

11. Nugent E, Neary P. Rectal cancer surgery: volume-outcome analysis. Int J Colorectal Dis. 2010;25:1389-96.

12. Borowski DW, Bradburn DM, Mills SJ, et al. Volume-outcome analysis of colerectal cancer-related outcomes. Br J Surg. 2010;97:1416-30.

13. Read TE, Myerson RJ, Fleshman JW, et al. Surgeon specialty is associated with outcome in rectal cancer treatment. Dis Colon Rectum. 2002;45;904-14.

14. Wexner SD, Rotholtz NA. Surgeon influenced variables in resectional rectal cancer surgery. Dis Colon Rectum. 2000;43: 1606-27.

15. Schlachta CM, Mamazza J, Seshadri PA et al. Defining a learning curve for laparoscopic colorectal resections. Dis Colon Rectum. 2001;44:217-22.

16. Tekkis P, Senagore AJ, Delaney CP, and Fazio VW. Evaluation of the learning curve in laparoscopic colorectal surgery: comparison of right-sided and left-sided resections. Ann Surg. 2005;242:83-91.

17. Dincler S, Koller MT, Steurer J et al. Multidimensional analysis of learning curves in laparoscopic sigmoid resection: eight-year results. Dis Colon Rectum. 2003;46:1371-8.

18. Wexner SD, Cohen SM, Ulrich A, Reissman P. Laparoscopic colorectal surgery: are we being honest with our patients? Dis Colon Rectum. 1995;38:723-7.

19. Miskovic D, Wyles SM, Ni M, et al. Systemic review on mentoring and simulation in laparoscopic colorectal surgery. Ann Surg. 2010;252:943-51.

20. Bashankaev B, Baido S, Wexner SD. Review of available methods of simulation training to facilitate surgical education. Surg Endosc. 2011;25:28-35.

21. Baik SH, Gincherman M, Mutch MG, et al. Laparoscopic vs. open resection for patients with rectal cancer: comparison of perioperative outcomes and long term survival. Dis Colon Rectum. 2011;54:6-14.

22. Buunen M, Bonjer HJ, Hop WC, et al. COLORII. A randomized clinical trial comparing laparoscopic and open surgery for rectal cancer. Dan Med Bull. 2009;56:89-91. 